\title{
Transcriptomic landscape of Pueraria lobata demonstrates potential for phytochemical study
}

\author{
Rongchun Han 1,2, Hiroki Takahashi', Michimi Nakamura', Naoko Yoshimoto',

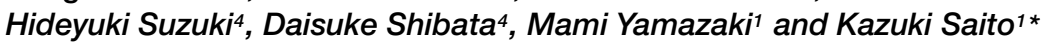

${ }^{1}$ Department of Molecular Biology and Biotechnology, Graduate School of Pharmaceutical Sciences, Chiba University, Chiba, Japan, ${ }^{2}$ Pharmacy College, Liaoning University of Traditional Chinese Medicine, Dalian, China, ${ }^{3}$ Medical Mycology Research Center, Chiba University, Chiba, Japan, ${ }^{4}$ Kazusa DNA Research Institute, Chiba, Japan

OPEN ACCESS

Edited by:

Robert L. Last,

Michigan State University, USA

Reviewed by:

LiTian,

University of California, Davis, USA

Guodong Wang,

Chinese Academy of Sciences, China

Alain Tissier,

Leibniz Institute of Plant Biochemistry,

Germany

*Correspondence: Kazuki Saito,

Department of Molecular Biology and Biotechnology, Graduate School of Pharmaceutical Sciences, Chiba University, Inohana 1-8-1, Chuo-ku,

Chiba 260-8675, Japan ksaito@faculty.chiba-u.jp

Specialty section: This article was submitted to Plant Metabolism

and Chemodiversity, a section of the journa Frontiers in Plant Science

Received: 14 April 2015 Accepted: 26 May 2015

Published: 22 June 2015

Citation:

Han R, Takahashi H, Nakamura M, Yoshimoto N, Suzuki H, Shibata $D$,

Yamazaki M and Saito K (2015)

Transcriptomic landscape of Pueraria lobata demonstrates potential

for phytochemical study.

Front. Plant Sci. 6:426.

doi: 10.3389/fpls.2015.00426
Pueraria lobata (Willd.) Ohwi has a long and broad application in the treatment of disease. However, in the US and EU, it is treated as a notorious weed. The information to be gained from decoding the deep transcriptome profile would facilitate further research on $P$. lobata. In this study, more than 93 million fastq format reads were generated by Illumina's next-generation sequencing approach using five types of $P$. lobata tissue, followed by CLC de novo assembly methods, ultimately yielding about 83,041 contigs in total. Then BLASTx similarity searches against the NCBI NR database and UniProtKB database were conducted. Once the duplicates among BLASTx hits were eliminated, ID mapping against the UniProt database was conducted online to retrieve Gene Ontology information. In search of the putative genes relevant to essential biosynthesis pathways, all 1,348 unique enzyme commission numbers were used to map pathways against the Kyoto Encyclopedia of Genes and Genomes. Enzymes related to the isoflavonoid and flavonoid biosynthesis pathways were focused for detailed investigation and subsequently, quantitative real-time reverse transcription polymerase chain reaction was conducted for biological validation. Metabolites of interest, puerarin and daidzin were studied by HPLC. The findings in this report may serve as a footstone for further research into this promising medicinal plant.

Keywords: Pueraria lobata, Leguminosae, Kudzu, deep-transcriptome analysis

\section{Introduction}

Pueraria lobata (Willd.) Ohwi (Kudzu) has been described and used as a traditional medicinal plant for more than 20 centuries in oriental medicine (Keung and Vallee, 1998). The P. lobata root, a part of the plant that is prescribed most frequently, accumulates abundant polyphenolic compounds, including isoflavones, isoflavonoid glycosides, coumarins, puerarols and the associated derivatives (Wong et al., 2011). Intensive investigation has revealed a chemical profile with antioxidant and antimutagenic activity (Miyazawa et al., 2001; Cherdshewasart and Sutjit, 2008) and efficacy in the treatment of alcoholism (Carai et al., 2000) and diabetic retinopathy (Cherdshewasart et al., 2007; Teng et al., 2009). The plant was introduced to the United States in 1876 as an ornamental plant and then to Europe. Due to its rapid growth and vigorous adaptation to the surroundings, $P$. lobata is now regarded as a major ecosystem threat (Follak, 2011) and a noxious weed, according to the USDA plant database. In order to evaluate the plant's potential as a cure for disease or regulate 
its invasive influence on other native plants, additional research using next-generation sequencing technologies into this leguminous plant is necessary.

The genomes for many model organisms have been sequenced but for these non-model plants, the lack of reference genome information hinders studies on the underlying genes involved in essential biological processes related to drug development. In this regard, transcriptomic sequencing plays an essential role in understanding the genetic diversity across organisms. Such approaches elucidate the genetic code that underlies protein diversity (Muranaka and Saito, 2013; Saito, 2013). The use of new technologies such as the Short Oligonucleotide Analysis Package (SOAPdenovo; Li et al., 2009), Assembly by Short Sequences (AbySS; Simpson et al., 2009), and Trinity (Grabherr et al., 2011) accelerates the pace of transcriptomic profiling when processing tremendous amounts of data generated from largescale sequencing projects. Massively parallel cDNA sequencing (RNA-Seq) measures the levels of transcripts and their isoforms far more precisely than other methods (Fullwood et al., 2009; Wang et al., 2009).

$C$-glycosides are widespread in plants, insects and microbes, where they serve a diverse range of functions including acting as antibiotics, antioxidants, attractants, and feeding deterrents (Brazier-Hicks et al., 2009). Early study on Fagopyrum esculentum seedlings showed 2-hydroxylation of flavanones was a critical prerequisite for the corresponding $C$-glucosyltransferase to catalyze (Kerscher and Franz, 1987). Recently, reports regarding $C$-glycosylation of flavonoids in crops such as wheat and corn suggested considerable similarity of the proteins some of which exhibited bifunctional $C$ - and $O$-glucosyltransferase activity (Ferreyra et al., 2013). For the characteristic compound daidzein8-C-glycoside (puerarin) found in $P$. lobata, although the biosynthetic pathway for daidzein in legumes is well established (Steele et al., 1999; Jung et al., 2000), the key enzyme responsible for catalyzing this isoflavone aglycon remains to be identified.

In this study, 93,248,914 paired-end reads of $P$. lobata were generated from five different tissues by Illumina's sequencing platform. Illumina reads were deposited at the DDBJ Sequence Read Archive (DRA) with accession number (AC) DRA001736 and the resultant contigs along with the top hits of BLASTx at GitHub ${ }^{1}$. CLC Genomics Workbench (CLC bio, Aarhus, Denmark) was subsequently applied to conduct de novo assembly. Based on the findings provided by Gene Ontology (GO) and KEGG pathway mapping, the candidate genes that may be involved in the biosynthesis of key chemical components were identified (Han et al., 2015). For biological validation, quantitative real-time reverse transcription polymerase chain reaction (qRT-PCR) was applied to check the genuine expression profile for the genes involved in the biosynthetic pathway leading to isoflavonoids. Meanwhile, the concentrations of puerarin and daidzin, the characteristic compounds in Kudzu, were measured by High Performance Liquid Chromatography (HPLC) within the five tissues from which we obtained the deep transcriptomic data.

${ }^{1}$ https://github.com/rongchunhan/Pueraria_lobata

\section{Materials and Methods}

\section{Plant Materials, Chemicals and Total RNA Extraction}

The basic technology of RNA extraction, RNA-seq analysis and informatics is similar as described elsewhere (Han et al., 2015). Fresh tissues and organs were collected from healthy P. lobata plants growing in Chiba, Japan in May 2012. Puerarin and daidzin standard substances were purchased from LC laboratories (USA). The materials were kept in RNA stabilization solution (RNAlater, Life technologies, USA) immediately after sampling. The RNAlater solution was gently removed with a Kimwipe, and the remaining sample was frozen by liquid nitrogen and powdered using Multi Beads Shocker (Yasui Kikai, Japan). TRIzol Reagent (Invitrogen, USA) was used to extract total RNA from powdered $P$. lobata. The RNA obtained was then treated using the RNeasy Mini Kit (Qiagen, USA).

\section{cDNA Library Preparation and Sequencing}

The TruSeq RNA Sample Prep Kit v2 (Illumina, CA, USA) was used for cDNA library preparation and sequencing. Once the mRNA in total RNA had been polyA-selected and fragmented, double-stranded cDNA was prepared for cDNA library construction. After the creation of bluntend fragments and indexed adaptor ligation, the samples were hybridized to flow cells. Cluster amplification was completed using the cBot Cluster Generation System (Illumina, CA, USA) and then sequenced by Illumina's next-generation sequencing instrument, the HiSeq 1000 as described (Han et al., 2015).

\section{CLC Approach to De Novo Assembly and Transcript Abundance Analysis}

Prior to assembly, the original fastq $P$. lobata format data were subjected to CLC trimming to eliminate reads of poor quality. The CLC method (version 4.9) was used to process clean reads. The publically available $P$. lobata expressed sequence tags (ESTs) data $(6,365)$ were downloaded from the National Center for Biotechnology Information (NCBI) database of expressed sequence tags (dbESTs). All resultant contigs over 200 bp were taken into consideration for the downstream analysis. Because the assembly process may lead to duplicate contigs, CD-HITEST was applied with representative sequences at $90 \%$ identity to obtain unique contigs (Fu et al., 2012).

In order to estimate contig expression level, we applied the CLC approach to map all fastq format reads back to the contigs and calculated the reads per kilobase of the transcript per million mapped reads (RPKM) values. Because the five samples lacked technical replicates, the non-parametric approach for the identification of differentially expressed genes, NOISeq-sim (Tarazona et al., 2011), was applied to analyze ten independent pair-wise sample comparisons.

\section{Annotation Pipeline and Data Mining}

As query sequences, all assembled contigs were subjected to the BLASTx sequence similarity search against the non-redundant (NR) protein database at NCBI and the Universal Protein 


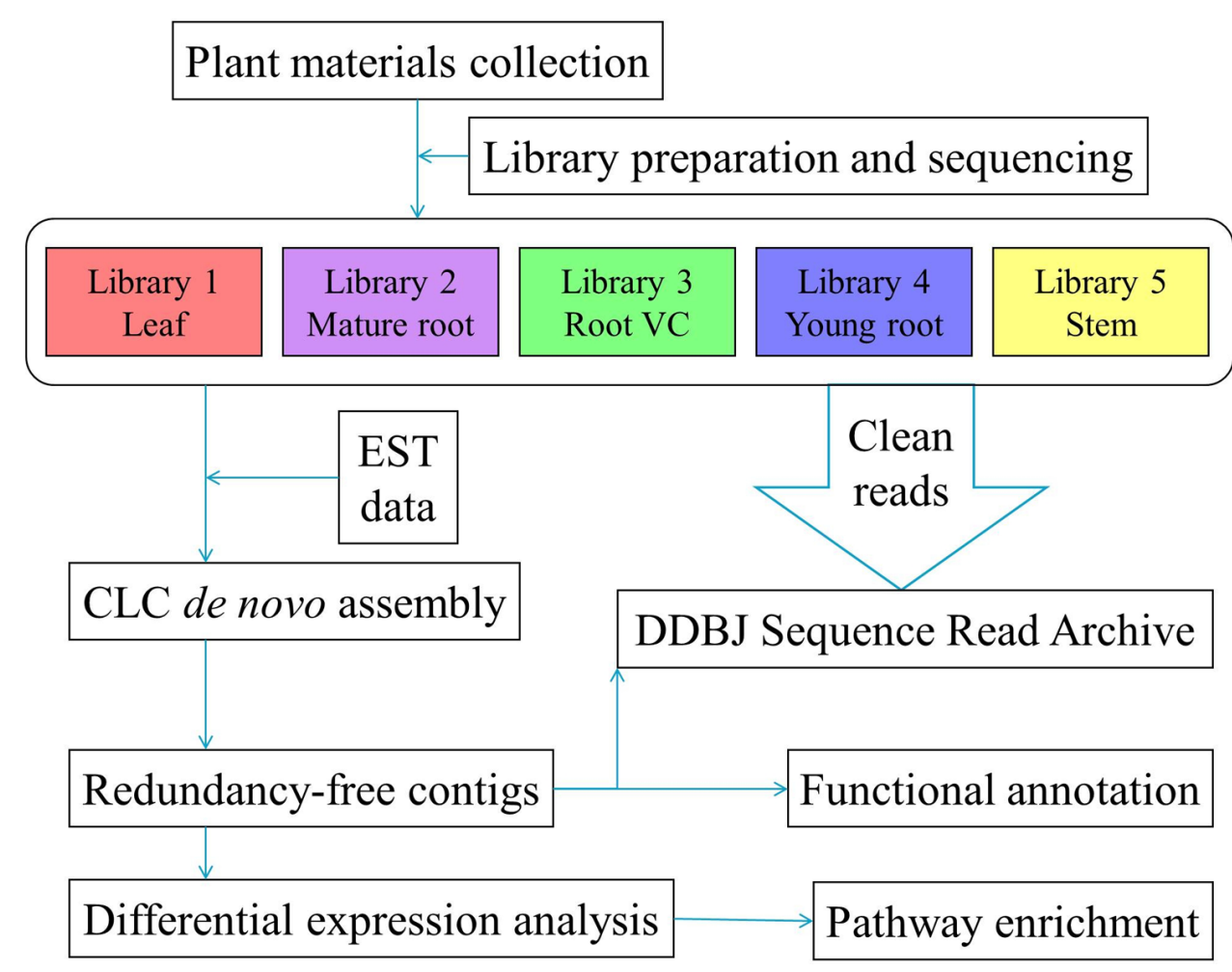

FIGURE 1 | Summary of the experimental design and analysis pipeline.

resource (UniProt) at UniProt consortium. The $e$-value threshold was set to $1 \mathrm{e}-10$; the upper limit on the number of subject sequences from databases to show alignment was limited to 20 . As to the large BLASTx output, only percent identities over $40 \%$ and $e$-values less than $1 \mathrm{e}-30$ were taken into consideration (Han et al., 2015). After eliminating redundancies, all unique gene identifiers in fasta format were then uploaded to the UniProt ID mapping website for online data processing ${ }^{2}$. By consolidating the returned target list and the UniProtKB ACs obtained from the above-mentioned BLASTx output against the UniProt database, we applied the redundancy-free ACs to annotation using the same online facilities. Out of the huge number of annotation results, we examined the reviewed findings from UniProtKB/Swiss-Prot as well as TrEMBL for data mining (Han et al., 2015). The contigs with GO terms at the protein level were classified. Ultimately, 1,348 enzyme commission (EC) numbers were collected to map pathways against KEGG, and the enzymes related to daidzein biosynthesis were depicted.

\section{Gene Expression Validation Adopting qRT-PCR}

Quantitative real-time reverse transcription polymerase chain reaction was conducted using 96-well plates and StepOnePlus real-time PCR system (Applied biosystems). Three technical replicates were used for each reaction, and a negative control consisting of template without primers was included for each

${ }^{2}$ http://www.uniprot.org template. Reaction volume was $15 \mu \mathrm{L}$ and each reaction comprised $7.5 \mu \mathrm{L}$ of SYBR Select Master mix $(2 \times), 0.15 \mu \mathrm{L}$ of $10 \mu \mathrm{M}$ primers (1:1 mix of forward and reverse primers), $1.0 \mu \mathrm{L}$ of cDNA synthesized using SuperScript VILO cDNA Synthesis Kit (Life technologies), and $6.35 \mu \mathrm{L}$ of nuclease-free distilled water. Reaction conditions included $10 \mathrm{~min}$ incubation at $95^{\circ} \mathrm{C}$,

TABLE 1 | Quality control for transcriptomic analysis.

\begin{tabular}{lllll}
\hline Library & $\begin{array}{l}\text { No. of reads } \\
\text { (paired) }\end{array}$ & $\begin{array}{l}\text { Average } \\
\text { length (bp) }\end{array}$ & $\begin{array}{l}\text { No. of reads } \\
\text { after trim (bp) }\end{array}$ & $\begin{array}{l}\text { Average length } \\
\text { after trim (bp) }\end{array}$ \\
\hline (1) Leaf & $18,247,136$ & 101.0 & $18,189,752$ & 98.8 \\
(2) Mature root & $14,090,648$ & 101.0 & $14,039,985$ & 98.2 \\
(3) Root VC & $20,847,896$ & 101.0 & $20,776,909$ & 98.7 \\
(4) Young root & $23,941,990$ & 101.0 & $23,869,723$ & 98.8 \\
(5) Stem & $16,429,912$ & 101.0 & $16,372,545$ & 98.5
\end{tabular}

TABLE 2 | Overview of $P$. lobata transcriptomic assembly.

\begin{tabular}{ll}
\hline Items & Numbers \\
\hline Total bases & $9,197,584,658$ \\
Average length of reads (bp) & 98.6 \\
No. of reads (6,365 ESTs included) & $93,255,279$ \\
Average length of contigs (bp) & 730 \\
N75; N50; N25 (bp) & $488 ; 1,145 ; 2,125$ \\
No. of contigs over 200 bp & 83,041 \\
Non-redundant contigs & 81,508
\end{tabular}




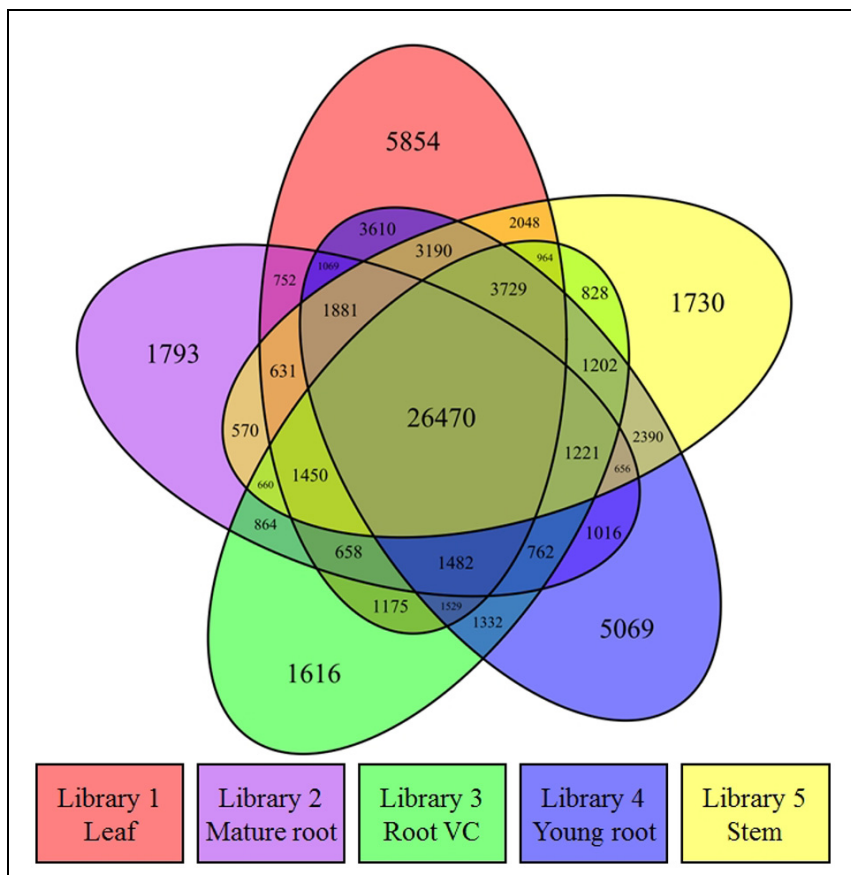

FIGURE 2 | Transcriptomic expression analysis. A Venn diagram shows the distribution of transcriptionally active contigs whose RPKM values are greater than 0 in at least one of the libraries.

then 40 cycles of $95^{\circ} \mathrm{C}$ for $15 \mathrm{~s}$ and $60^{\circ} \mathrm{C}$ for $1 \mathrm{~min}$, followed by a melt-curve analysis to confirm single PCR product amplification. $\beta$-actin was used as the internal control gene (Hong et al., 2010). No amplification was observed in any negative control. Equivalent slopes for target and internal control gene were observed in amplification plots, so the comparative thresholdcycle $\left(\mathrm{C}_{\mathrm{T}}\right)$ method was used to calculate relative expression levels as $2^{-\Delta \mathrm{Ct}}$ where $\Delta \mathrm{Ct}=\left(\mathrm{C}_{\mathrm{T}}\right.$ target gene $-\mathrm{C}_{\mathrm{T}}$ internal control gene), assuming similar PCR efficiencies of target and internal control gene (Schmittgen and Livak, 2008; Gaines et al., 2014).

\section{HPLC Analysis of Puerarin and Daidzin in Five Tissues of $P$. lobata}

One gram of every fresh tissue studied was ground to powder in liquid nitrogen and extracted overnight with $5 \mathrm{ml}$ of acetone at $4^{\circ} \mathrm{C}$. Then the extract was centrifuged at 3,500 rpm for $30 \mathrm{~min}$ (He et al., 2008, 2011) and the supernatant was dried in ventilator. The residues were resuspended in methanol, and $10 \mu \mathrm{L}$ of the solution was analyzed by reverse-phase HPLC (HITACHI D7000 system) on a $5-\mu \mathrm{m} \mathrm{C15}$ column $(150 \times 4.6 \mathrm{~mm}$, Mightysil $)$. The flow rate was $0.5 \mathrm{~mL} / \mathrm{min}$ with the mobile phase methanol/water (25:75).

\section{Results}

\section{Plant RNA Extraction and cDNA Library Preparation}

Studies on legumes showed the isoflavones daidzein and genistein were major metabolites in all embryonic organs within the dry seeds. Seedling roots and callus cultures are known to produce daidzein, with the highest daidzein concentration to be found in mature fruits (Graham, 1991; Bouque et al., 1998). We intended collecting information regarding the nature of the genes responsible for the biosynthesis of daidzein and daidzin in P. lobata. We extracted total RNA from the leaf, mature root, root vascular cylinder (Root VC), young root and stem of the plant. Five distinct cDNA libraries were established from these five tissue samples. We will refer to the five libraries in the following manner: Library 1 (leaf), Library 2 (mature root), Library 3 (root VC), Library 4 (young root), Library 5 (stem). Wherever applicable, a uniform color scheme will be used to represent the libraries: red (Library 1), purple (Library 2), green (Library 3), blue (Library 4 ), and yellow (Library 5 ).

\section{Illumina Sequencing and De Novo Assembly}

All five libraries were processed using the Illumina HiSeq 1000 platform. Reads of poor quality, empty reads and those with unknown bases were trimmed by CLC software. In order to consolidate the available bio-information to obtain more reliable and thorough findings, we combined the resultant clean reads with $P$. lobata EST sequences obtained from the NCBI database to conduct de novo assembly; thus, 83,041 contigs were generated. By adopting CD-HIT-EST with a threshold set to 0.9 , duplicates were retrieved and discarded, leaving 81,508 NR contigs for downstream analysis. An overview of the experimental pipeline is shown in Figure 1. Tables 1 and 2 summarize trimming, sequencing, and assembly results.

\section{Guanine-Cytosine (GC) Content Analysis}

The reported GC content for unigene sequences in soybean and Arabidopsis is 0.43 and 0.44, respectively (Tian et al., 2004; Kawaguchi and Bailey-Serres, 2005). The mean GC content of P. lobata transcripts was found to be $39.9 \%$ (Supplementary S1). In eukaryotes, average GC content covers the range from $\sim 20$ to $60 \%$ (Serres-Giardi et al., 2012). Our values are in the middle of this range, slightly lower than those reported for Glycine max (43\%) but very close to those reported for Sophora flavescens (39.3\%) and Medicago truncatula (40\%) (Tian et al., 2004; Han et al., 2015).

\section{Transcriptome Information and Differential Accumulation of Transcripts}

RPKM calculation was conducted for transcript expression analysis after RNA-seq reads from each library were aligned to all contigs. A Venn diagram was drawn by utilizing the $\mathrm{R}$ project in conjunction with the VennDiagram package (Chen and Boutros, 2011) to illustrate the distribution profile of all active contigs $(78,201)$ with RPKM values $>0$ in at least one of the libraries (Figure 2). 33.8\% of the active transcripts were expressed across all five libraries, suggesting the homogeneity and high quality of the acquired raw data. Compared to other three tissues, young root and leaf had more exclusively expressed contigs, which demonstrated in spring, such tissues played important and unique physiological roles. RPKM calculation considers gene length variation and the number of total mapped reads, 


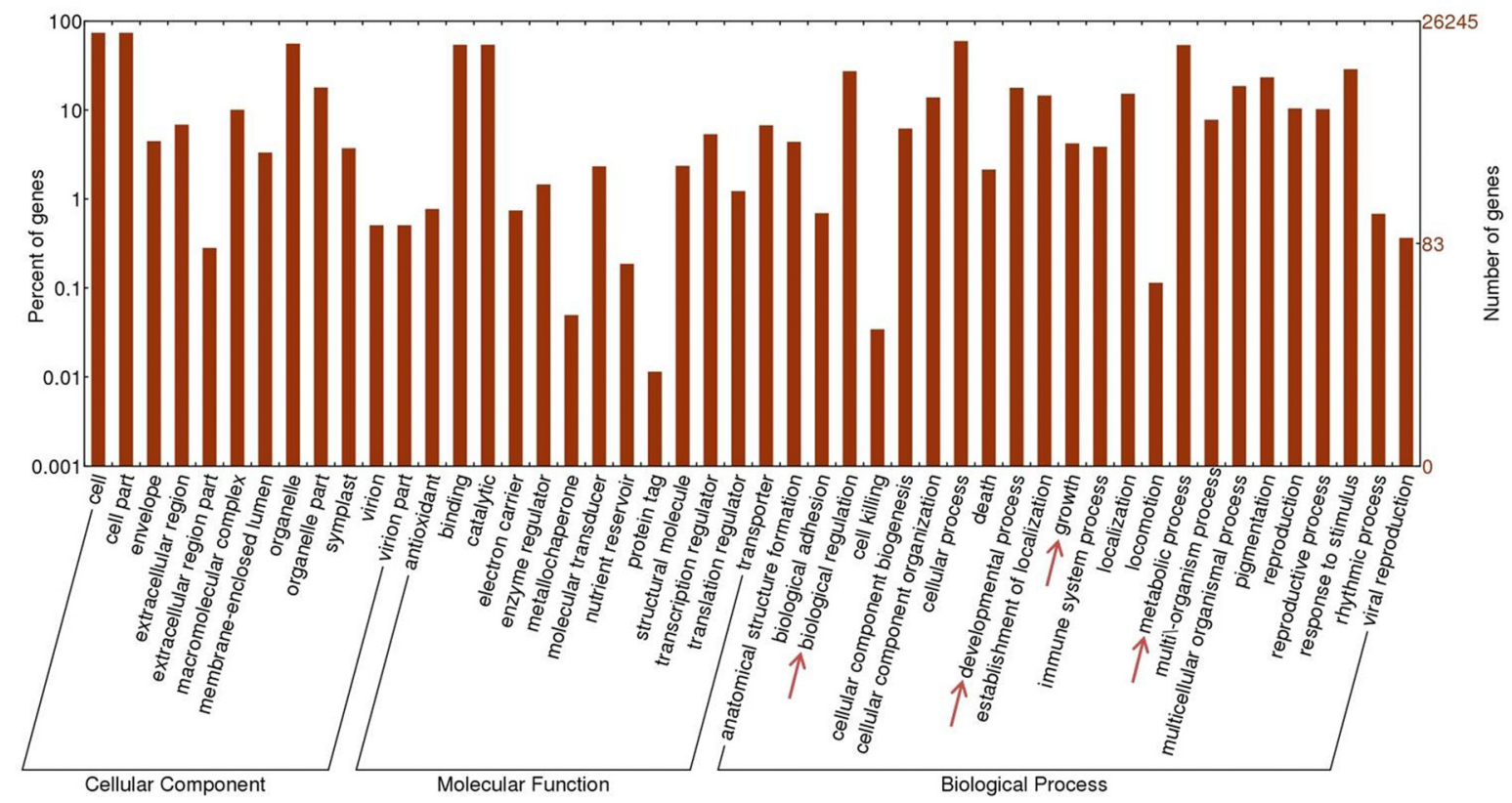

FIGURE 3 | Gene Ontology annotation for $\boldsymbol{P}$. lobata contigs. Forty eight subcategories are affiliated to three main domains: molecular function, cellular components and biological processes.

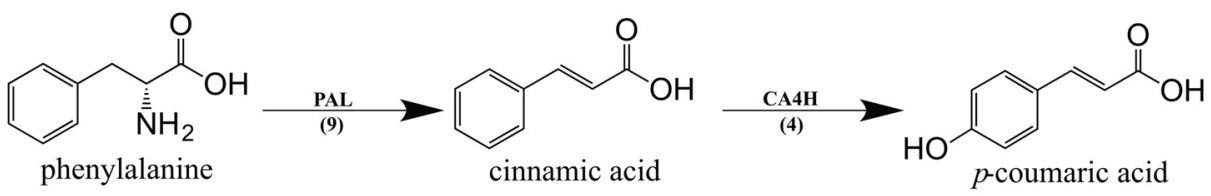

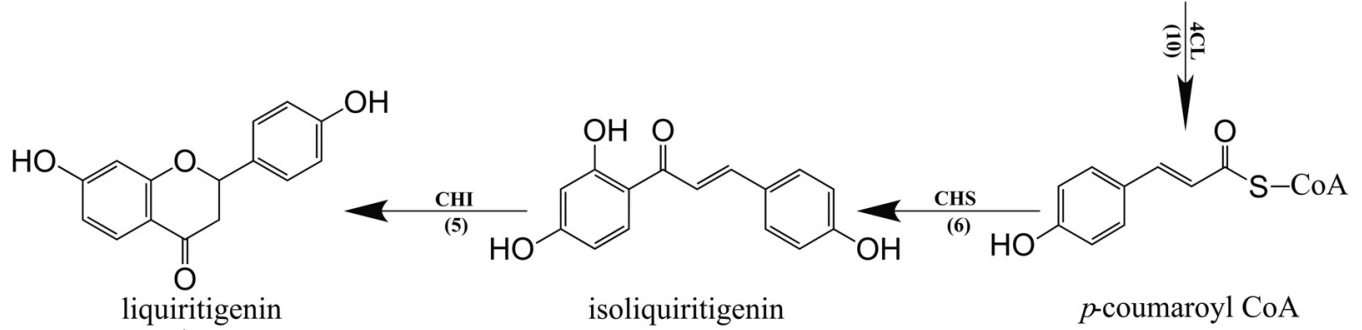<smiles>O=C1c2ccc(O)cc2OC(O)C1c1ccc(O)cc1</smiles>

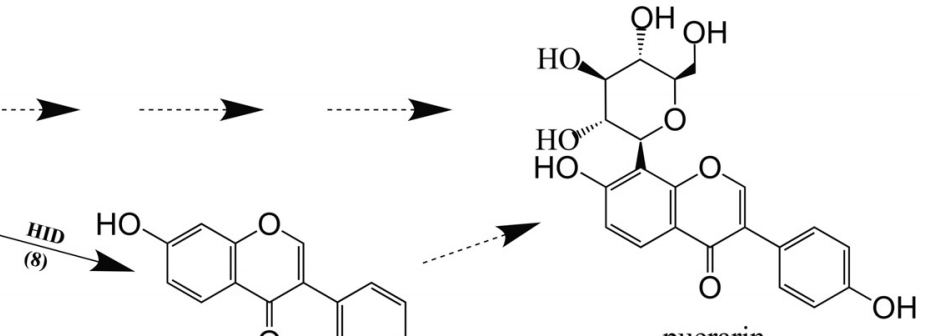

daidzein

FIGURE 4 | Proposed daidzein biosynthesis pathway in $P$. lobata. Every enzyme is followed by the number of corresponding contigs in parentheses. PAL, Phenylalanine ammonia-lyase, EC 4.3.1.24; CA4H, Trans-cinnamate 4-monooxygenase, EC 1.14.13.11; 4CL, 4-coumarate-CoA ligase, EC 6.2.1.12; CHS, 6'-deoxychalcone synthase,
EC 2.3.1.170; $\mathrm{CHI}$, Chalcone isomerase, EC 5.5.1.6; IFS, 2-hydroxyisoflavanone synthase, EC 1.14.13.136; HID,

2-hydroxyisoflavanone dehydratase, EC 4.2.1.105. Solid arrows show the pathways identified by the data obtained while the dotted arrows show unsolved steps. 


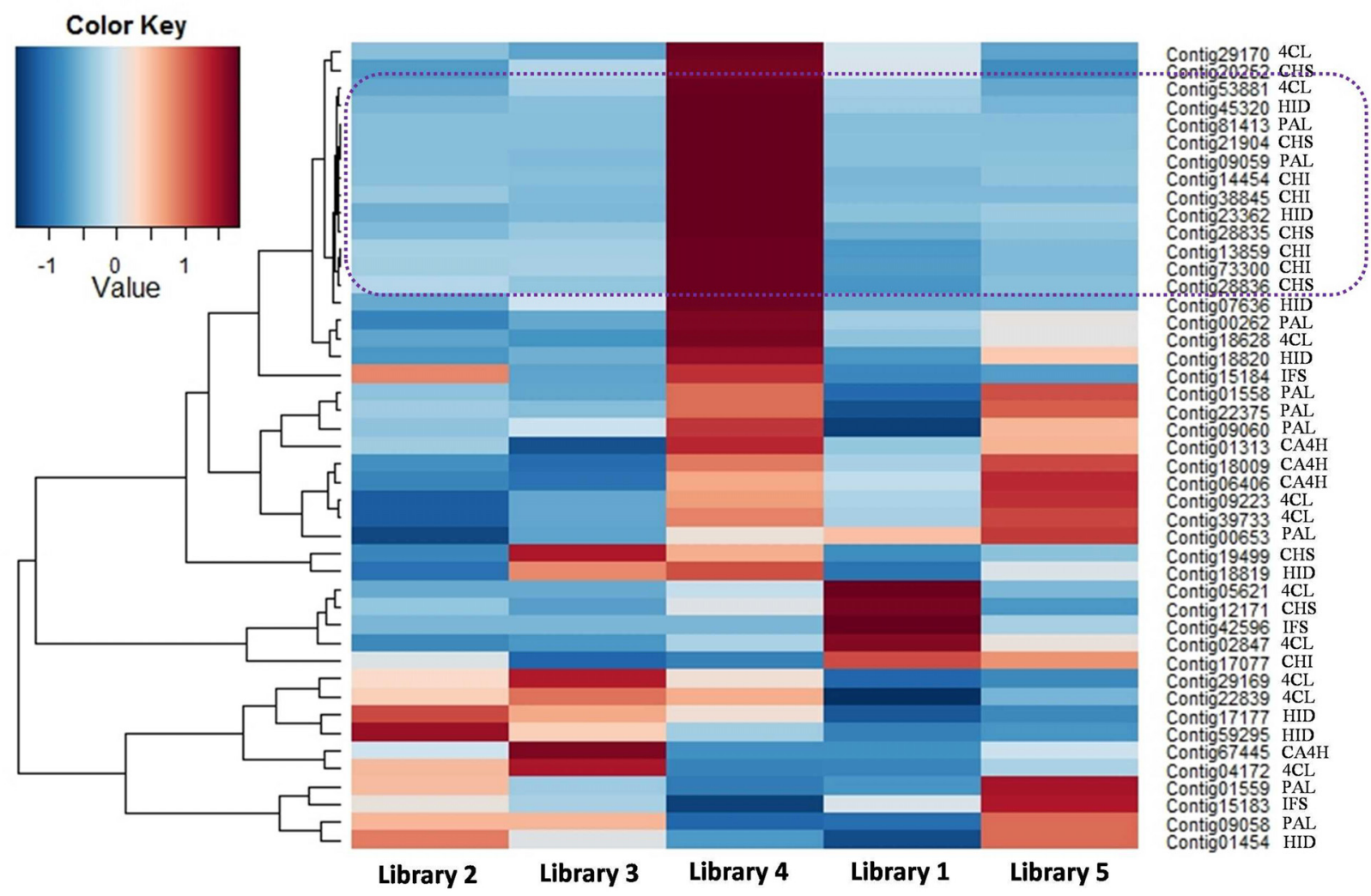

FIGURE $\mathbf{5}$ | Heatmap showing the expression profile for $\mathbf{4 5}$ contigs related to daidzein biosynthesis. Next to each contig name, the enzyme abbreviation is presented.

TABLE 3 | Validation of differentially expressed genes related to isoflavonoid biosynthesis.

\begin{tabular}{|c|c|c|c|c|c|c|c|c|c|}
\hline \multirow[t]{3}{*}{ Contig } & \multirow[t]{3}{*}{ Annotation } & \multicolumn{8}{|c|}{ Fold change : RNA-Seq and qRT-PCR validation } \\
\hline & & \multicolumn{2}{|c|}{ Lib 2/Lib 1} & \multicolumn{2}{|c|}{ Lib 4/Lib 1} & \multicolumn{2}{|c|}{ Lib 3/Lib 1} & \multicolumn{2}{|c|}{ Lib 5/Lib 1} \\
\hline & & RPKM & $2^{-\Delta C t}$ & RPKM & $2^{-\Delta C t}$ & RPKM & $2^{-\Delta C t}$ & RPKM & $2^{-\Delta C t}$ \\
\hline 21904 & $\mathrm{CHS}$ & 0.49 & 1.87 & $48^{*}$ & 36 & 0.76 & 0.97 & 0.47 & 0.60 \\
\hline 14454 & $\mathrm{CHI}$ & 1.20 & 0.72 & 9.0 & 14.6 & 1.12 & 0.63 & 1.29 & 2.07 \\
\hline 15184 & IFS & 20.1 & 2.90 & 25.1 & 6.84 & 3.31 & 2.45 & 2.69 & 2.14 \\
\hline 01454 & HID & 5.87 & 7.86 & 2.27 & 12.3 & 4.04 & 8.51 & 6.07 & 25.1 \\
\hline
\end{tabular}

Fold change of RPKM and $2^{-\Delta \mathrm{Ct}}$ between Lib 1 (Library 1) and other Libraries, respectively. *differently expressed gene detected by NOISeq-sim. CHS chalcone synthase, CHI Chalcone isomerase, IFS 2-hydroxyisoflavanone synthase, HID 2-hydroxyisoflavanone dehydratase.

which allows this normalized output to be used directly for the comparison of gene expression. The transcript RPKM values which are greater than 0 in at least one of the libraries are listed in Supplementary S2. To perform differential expression (DE) analysis, 10 pair-wise comparisons for the five libraries were conducted by applying NOISeq-sim, which is a non-parametric approach for the identification of differentially expressed genes from count data or previously normalized count data. By running NOISeq-sim on an R language platform with the given threshold $(q=0.9)$ for selecting differentially expressed features, the resultant number of DE transcripts varied across comparisons (Han et al., 2015). The highest value obtained was 1,408 differences between leaf and mature root transcripts; the lowest value obtained was 297 differences between mature root and root vascular cylinder (Supplementary S3).

\section{Protein Function Annotations and Gene Ontology Classification}

Functional annotations according to sequence similarity are often the initial step in studying the role and biological functions of gene products (Ramilowski et al., 2013). The basic local alignment search tool (BLAST) was utilized to scan nucleotide query sequences against protein databases (NR, UniProt) to identify similar subject sequences. When the threshold $e$-value 
TABLE 4 | Putative glucosyltransferases with Pearson correlation coefficients to HID and IFS.

\begin{tabular}{|c|c|c|c|}
\hline Contig_ID & $\begin{array}{c}\text { Coefficient } \\
\text { _to_HID }\end{array}$ & $\begin{array}{c}\text { Coefficient } \\
\text { _to_IFS }\end{array}$ & Annotation \\
\hline Contig02990 & 0.37 & 0 & Sucrose-UDP glucosyltransferase \\
\hline Contig03131 & -0.4 & 0.74 & Anthocyanidin 3-O-glucosyltransferase \\
\hline Contig03133 & -0.45 & 0.48 & Anthocyanidin 3-O-glucosyltransferase \\
\hline Contig05593 & -0.59 & 0.53 & Glycoprotein glucosyltransferase \\
\hline Contig09646 & -0.68 & 0.31 & Cytokinin-O-glucosyltransferase \\
\hline Contig10691 & -0.59 & 0.45 & glycoprotein glucosyltransferase \\
\hline Contig11620 & 0.79 & 0.1 & Putative glucosyltransferase \\
\hline Contig11621 & -0.69 & -0.4 & Putative glucosyltransferase \\
\hline Contig11622 & -0.84 & -0.39 & Putative glucosyltransferase \\
\hline Contig12257 & -0.66 & -0.54 & Cytokinin-O-glucosyltransferase \\
\hline Contig14425 & -0.77 & 0.47 & Putative UDP-glucosyltransferase \\
\hline Contig15137 & -0.6 & 0.54 & glycoprotein glucosyltransferase \\
\hline Contig15603 & -0.72 & -0.47 & Isoflavonoid glucosyltransferase \\
\hline Contig20530 & -0.78 & -0.3 & Sterol 3- $\beta$-glucosyltransferase \\
\hline Contig22923 & -0.07 & 0.2 & Sterol 3- $\beta$-glucosyltransferase \\
\hline Contig22924 & -0.94 & 0.04 & Sterol 3- $\beta$-glucosyltransferase \\
\hline Contig23483 & -0.4 & 0.73 & Zeatin O-glucosyltransferase \\
\hline Contig23956 & -0.35 & 0.79 & Isoflavonoid glucosyltransferase \\
\hline Contig25035 & -0.19 & 0.94 & Putative glucosyltransferase \\
\hline Contig28030 & -0.29 & -0.39 & flavonoid 3-O-glucosyltransferase \\
\hline Contig28462 & -0.03 & 0.46 & Hydroquinone glucosyltransferase \\
\hline Contig29838 & -0.47 & 0.22 & Isoflavone 7-O-glucosyltransferase 1 \\
\hline Contig29839 & 0.15 & -0.43 & Isoflavone 7-O-glucosyltransferase 1 \\
\hline Contig31158 & 0.34 & -0.12 & Cytokinin-O-glucosyltransferase \\
\hline Contig32270 & -0.39 & 0.71 & Anthocyanidin 3-O-glucosyltransferase \\
\hline Contig32277 & -0.13 & 0.58 & Hydroquinone glucosyltransferase \\
\hline Contig40732 & -0.67 & 0.07 & Sucrose-UDP glucosyltransferase \\
\hline Contig42441 & -0.9 & -0.18 & Cytokinin-O-glucosyltransferase \\
\hline Contig43033 & 0.27 & -0.7 & Isoflavone 7-O-glucosyltransferase 1 \\
\hline Contig44304 & -0.15 & 0.59 & Isoflavone 7-O-glucosyltransferase 1 \\
\hline Contig45936 & -0.04 & -0.98 & Isoflavone 7-O-glucosyltransferase 1 \\
\hline Contig46490 & 0.34 & -0.55 & Anthocyanidin 3-O-glucosyltransferase \\
\hline Contig47890 & -0.28 & 0.75 & Sterol 3- $\beta$-glucosyltransferase \\
\hline Contig51492 & -0.41 & 0.7 & UDP-glucosyltransferase \\
\hline Contig52569 & -0.22 & 0.64 & Sterol 3- $\beta$-glucosyltransferase \\
\hline Contig65011 & -0.4 & 0.68 & UDP-glucosyltransferase \\
\hline Contig73434 & 0.56 & -0.38 & Limonoid UDP-glucosyltransferase \\
\hline Contig74493 & 0.1 & 0.21 & Anthocyanidin 3-O-glucosyltransferase \\
\hline Contig77022 & -0.35 & 0.47 & Anthocyanidin 3-O-glucosyltransferase \\
\hline Contig79587 & -0.4 & 0.73 & Cytokinin-O-glucosyltransferase \\
\hline Contig79864 & -0.4 & 0.73 & Cytokinin-O-glucosyltransferase \\
\hline Contig03787 & 0.47 & -0.6 & Glucosyltransferase \\
\hline Contig06374 & -0.83 & 0.07 & Glucosyltransferase-13 (Fragment) \\
\hline Contig11423 & -0.71 & -0.49 & Glucosyltransferase \\
\hline Contig14082 & -0.58 & -0.42 & Glucosyltransferase-2 \\
\hline Contig14083 & -0.6 & -0.35 & Glucosyltransferase-12 \\
\hline Contig14085 & 0.3 & 0.89 & Glucosyltransferase-2 \\
\hline Contig24631 & 0.04 & 0.67 & Glucosyltransferase- 5 \\
\hline Contig28995 & -0.33 & 0.73 & Glucosyltransferase-12 \\
\hline
\end{tabular}

for BLASTx searches was set to $1 \mathrm{e}-10$ and the top 20 subject sequences for each query sequence were taken into consideration, we obtained 829,087 subject sequences for all 81,508 query sequences. To obtain reliable results while reducing redundancy, we set stricter requirements for retrieving the candidate genes (Han et al., 2015). With this approach, significant matches were assigned to 30,156 contigs.

Gene Ontology, comprising domains of biological processes, molecular functions and cellular components, is a useful instrument with which to study the nature of annotated genes (Ashburner et al., 2000). With the help of Web Gene Ontology Annotation Plot (WEGO) software (Ye et al., 2006), 26,245 contigs yielded corresponding GO terms that could be further classified into 48 sub-categories: 12 related to cellular components, 13 to molecular function and 23 to biological processes. Figure 3 presents the large number of transcripts related to metabolic processes $(14,164)$ and biological regulation $(7,194)$. The contigs assigned to "growth" and "developmental process" under GO biological process may hold the key to the mechanism underlying its rapid and aggressive growth rate. The disadvantage of evaluating gene classification by directly counting the number of GO terms which possess the same or very similar functions is that the expression level of these query sequences varies, which grants distinct weight to the same GO term as it corresponds to different query sequences. With this concern, overrepresented GO terms were identified by Fisher's exact test. The one-tailed Fisher's exact $p$-values corresponding to overrepresented categories were calculated according to the counts in $2 \times 2$ contingency tables. Counts $n_{11}, n_{12}, n_{21}$, and $n_{22}$ in each table stand for: $n_{11}$, number of observations of a specific category in the first gene set; $n_{12}$, number of other categories in the first gene set; $n_{21}$, number of observations of a category in the second gene set; and $n_{22}$, number of observations of other categories in the second gene set (Takahashi et al., 2011; Han et al., 2015). p-values were corrected utilizing the false discovery rate (FDR) method (Benjamini and Hochberg, 1995) with the threshold of 0.05 . For each $P$. lobata library, contigs with RPKM value over 15.0 (the top $\sim 10 \%$ of all transcripts) were regarded as highly expressed genes and extracted respectively. Then the merged 14,364 contigs were used to perform Fisher's exact test.

As with many woody vines supported by trees or man-made structures, Kudzu may allocate the majority of its biomass to vine elongation and leaf growth. It is regarded as an invasive alien plant in Europe and northern America because its rapid growth rate (up to $30 \mathrm{~cm} \mathrm{~d}^{-1}$; Lindgren et al., 2013) allows Kudzu to suffocate neighboring plants that are deprived of sunlight. The overrepresented GO terms (Supplementary S4, GO terms with $p<1 \mathrm{E}-30$ are listed) suggest that highly activated biological processes such as cell division (GO: 0051301), cell growth (GO: 0016049), root hair elongation (GO: 0048767), response to cold (GO: 0009409) and response to salt stress (GO: 0009651) could play an essential role in its aggressive development.

\section{KEGG Pathway Retrieval}

The Kyoto Encyclopedia of Genes and Genomes (KEGG) provides a robust instrument for biological pathway assignment 
as well as the functional annotation of gene products (Han et al., 2015). From ID mapping results, we obtained 1,348 unique enzymes corresponding to 16,380 contigs and subsequently retrieved pathways using KEGG. The EC numbers were assigned to 152 biological pathways with more than half of the enzymes (697) involved in metabolic pathways. Regarding the significant capacity of leguminous plants to accumulate functional flavonoids, 19 flavonoid biosynthetic and 14 isoflavonoid biosynthetic enzymes are presented in Supplementary S5.

\section{Genes Involved in Isoflavonoid Biosynthesis in P. lobata}

Flavonoids are a group of polyphenolic compounds distributed widely throughout the plant kingdom. These compounds modulate the activity of enzymes to benefit the entire organism. As an important subgroup of flavonoids, isoflavonoids are mainly produced in legumes and affect oxidative stress markers, immune function and adipogenesis (Miadokova, 2009; Han et al., 2015).

In the phenylpropanoid pathway, the synthesis of flavonoids is initialized by transforming phenylalanine into $p$-coumaroylCoA. To initiate flavonoid biosynthesis, chalcone synthase catalyzes the formation of chalcone scaffolds, from which all flavonoids derive (Falcone Ferreyra et al., 2012; Saito et al., 2013; Han et al., 2015). Based on our functional annotation findings, 45 contigs were predicted to represent seven enzymes critical to the biosynthesis of daidzein, which may be necessary to make the puerarin found in P. lobata. The number of contigs corresponding to each enzyme and the biosynthetic pathway are presented in Figure 4. Figure 5 shows the expression profile for the 45 contigs corresponding to seven daidzein-biosynthesis-related enzymes across 5 libraries. From the heatmap, majority of the contigs had strong expression in library 4 (young root) and high correlation could be found among phenylalanine ammonia-lyase (PAL), 4-coumarate-CoA ligase (4CL), 6'-deoxychalcone synthase (CHS), chalcone isomerase (CHI) and 2-hydroxyisoflavanone dehydratase (HID), which gave light to the biosynthetic pathway of flavonoids in P. lobata. A previous study (Chen et al., 2001) showed that concentrations of puerarin and daidzein were up to threefold higher in the roots as compared to the veins of P. lobata. In our study, biosynthesis of the chalcone scaffold showed that the expression of downstream enzymes exhibits a clear pattern that provides evidence into the organ-specific biosynthesis of daidzein (Supplementary S6). In $P$. lobata, early steps for the biosynthesis of daidzein and even puerarin might take place mainly in young root. After transportation of the required precursors to other parts of the plant or along with organ growth, the expression of related enzymes increases in mature root. Finally, the high expression of 2-hydroxyisoflavanone dehydratase in stem and mature root may account for the accumulation of daidzein and puerarin in certain plant organs.

Figure 4 demonstrates 45 contigs involved in the isoflavonoid biosynthetic pathway. We carried out to validate the genuine biological expression profile of such contigs, focusing on the genes from chalcone synthase (EC 2.3.1.170) to 2hydroxyisoflavanone dehydratase (EC 4.2.1.105) since these are crucial factors leading to the accumulation of isoflavonoids. According to Supplementary S6, several contigs can be aligned to each of the four enzymes. Therefore, we set the following criteria for selecting the appropriate candidate contigs in qRTPCR experiment: firstly, the contig itself should consist of long fragment; secondly, the expression judging by RPKM values across the five tissues was relatively high; thirdly, the identity between the candidate contig and target enzyme should be high. Contig 21904, 14454, 15184, and 01454, corresponding to chalcone synthase, chalcone isomerase, 2-hydroxyisoflavanone synthase and 2-hydroxyisoflavanone dehydratase, respectively, were chosen to perform qRT-PCR. For CHS and CHI, Table 3 shows similar pattern of gene expression profile between deep transcriptomic data and qRT-PCR result. Regarding chalcone synthase, its DE between young root and leaf was detected by NOISeq-sim and the ratio of $2^{-\Delta \mathrm{Ct}}$ was also the highest one, 36. However, although the trend that the expression of 2hydroxyisoflavanone synthase (IFS) and HID in library 1 was always lower compared to other libraries was verified by both approaches, the ratio varied. For example, RPKM value suggested 20.1-fold elevated expression in library 2 while qRT-PCR granted only 2.90 -fold. We collected the samples for sequencing in 2012 but the materials used for qRT-PCR were obtained in 2014 from the same location, though. The slightly changed sampling conditions may result in the variations in validation experiment. The primers designed for qRT-PCR are listed in Supplementary S7.

\section{Discussion}

From the expression pattern of isoflavonoid biosynthesis in $\mathrm{Kudzu}$, transportation of certain precursors into other parts of the plant for downstream reaction may be required (refer to genes involved in isoflavonoid biosynthesis in $P$. lobata). By searching the annotation data, 41 expressed ABC transporters were retrieved. Regarding IFS and HID, suggested by the changes of expression level in different tissues, transportation of the intermediates may occur. Supplementary S8 lists 41 $\mathrm{ABC}$ transporters found in Kudzu dataset along with Pearson correlation coefficients to contig 15184 which is a putative IFS and contig 01454, an annotated HID.

TABLE 5 | Determination of puerarin and daidzin in fresh plant samples.

\begin{tabular}{|c|c|c|c|c|c|}
\hline & Leaf & Stem & Mature root & Young root & Root VC \\
\hline Daidzin & N.D. & N.D. & $0.668 \pm 0.004$ & $0.156 \pm 0.002$ & $0.994 \pm 0.008$ \\
\hline
\end{tabular}

a N.D. = Not detected. ${ }^{\mathrm{b}}$ Means $\pm S D(\mathrm{mg} / \mathrm{g}$ fresh weight, $n=3)$. 
A recent study provided fairly constructive insights into the biosynthetic pathway of puerarin and contributed more than 6,365 ESTs (He et al., 2011). We integrated the publically available ESTs into our raw reads from five different tissues of Kudzu and then performed de novo assembly altogether. This enabled us to utilize related information and the assembled contigs showed identical or highly similar transcripts with the ESTs regarding glucosyltransferase.

Many $C$-glucosyltransferases have been identified in bacteria, insects and plants, especially in cereals. The elucidated mechanism for $C$-glycosylation of flavonoids proved 2hydroxylation of flavanones was the appropriate premise for the catalytic reaction to proceed. Likewise, in studying the biosynthesis of puerarin, 2-hydroxylation of isoflavanone $\left(2,7,4^{\prime}\right.$ trihydroxy-isoflavanone) should be considered as a possible substrate for its formation when daidzein as a direct putative precursor meets with obstacles (Figure 4). With the formation of trihydroxy-isoflavone 8 - $C$ glycoside catalyzed by suitable UDP-dependent glucosyltransferases, the glycoside may be subjected to dehydration reaction, resulting in puerarin. Forty nine contigs were annotated as glucosyltransferase in our dataset, if the target glucosyltransferase utilizes either one of the above-mentioned precursors, the correlation with the enzyme directly producing 2,7,4'-trihydroxy-isoflavanone or daidzein would be significant. Table 4 lists the annotated glucosyltransferases along with Pearson correlation coefficients to HID and IFS.

Kudzu root, which is the main part prescribed in oriental medicines in treating various diseases, produce predominantly isoflavone $C$ - and $O$-glucosides. We collected five tissues from which the deep transcriptomic data were generated and studied puerarin and daidzin profile using HPLC. Both puerarin and daidzin are highly accumulated in mature root and root vascular cylinder and the concentration of puerarin is higher than that of daidzin (Table 5, Supplementary S9). Although several genes related to isoflavonoid biosynthesis are highly expressed in young root, the concentration of the two compounds is low in young root compared with that in mature root. This may be due to the essential enzymes for the production of puerarin actively expressed in the young and mature roots but the

\section{References}

Ashburner, M., Ball, C. A., Blake, J. A., Botstein, D., Butler, H., Cherry, J. M., et al. (2000). Gene ontology: tool for the unification of biology. The Gene Ontology Consortium. Nat. Genet. 25, 25-29. doi: 10.1038/ 75556

Benjamini, Y., and Hochberg, Y. (1995). Controlling the false discovery rate - a practical and powerful approach to multiple testing. J. R. Stat. Soc. B Methodol. 57, 289-300.

Bouque, V., Bourgaud, F., Nguyen, C., and Guckert, A. (1998). Production of daidzein by callus cultures of Psoralea species and comparison with plants. Plant Cell Tissue Organ Cult. 53, 35-40. doi: 10.1023/A:1006057211490

Brazier-Hicks, M., Evans, K. M., Gershater, M. C., Puschmann, H., Steel, P. G., and Edwards, R. (2009). The C-glycosylation of flavonoids in cereals. J. Biol. Chem. 284, 17926-17934. doi: 10.1074/jbc.M109.009258

Carai, M. A., Agabio, R., Bombardelli, E., Bourov, I., Gessa, G. L., Lobina, C., et al. (2000). Potential use of medicinal plants in the treatment of accumulation of puerarin does not reach to the maximum yet in the young root. Deep transcriptomic data obtained in this study may provide the key to this question. In relatively young stem which was used in this study and leaf, daidzin was not detectable.

RNA-Seq analysis is cost effective and the most efficient approach currently available to manage high-throughput data. By consolidating data information obtained from five P. lobata libraries, we analyzed the DE profile and mapped biosynthetic pathways against KEGG using enzyme ACs. Evaluating overrepresented GO terms by considering the RPKM values of the corresponding contigs provided a more accurate representation of the data. By qRT-PCR and HPLC, both gene expression validation and metabolite analysis were performed. The deep transcriptomic data we present here may facilitate future research on this promising plant.

\section{Author Contributions}

KS and MY designed the research framework. MN conducted total RNA extraction. HS and DS performed deep-transcriptome sequencing. RH, HT conducted the data interpretation and laboratory analysis. RH, KS and NY contributed to the manuscript preparation.

\section{Acknowledgments}

This study was supported, in part, by Grants-in-Aid for scientific research from the Ministry of Education, Culture, Sports, Science and Technology (MEXT) of Japan and by Health Labour Sciences Research Grant from the Ministry of Health, Labour and Welfare, Japan.

\section{Supplementary Material}

The Supplementary Material for this article can be found online at: http://journal.frontiersin.org/article/10.3389/fpls.2015.00426

alcoholism. Fitoterapia 71, S38-S42. doi: 10.1016/S0367-326X(00) 00178-7

Chen, G., Zhang, J., and Jiannong, Y. (2001). Determination of puerarin, daidzein and rutin in Pueraria lobata (Wild.) Ohwi by capillary electrophoresis with electrochemical detection. J. Chromatogr. A 923, 255-262. doi: 10.1016/S00219673(01)00996-7

Chen, H., and Boutros, P. C. (2011). VennDiagram: a package for the generation of highly-customizable Venn and Euler diagrams in R. BMC Bioinform. 12:35. doi: 10.1186/1471-2105-12-35

Cherdshewasart, W., Subtang, S., and Dahlan, W. (2007). Major isoflavonoid contents of the phytoestrogen rich-herb Pueraria mirifica in comparison with Pueraria lobata. J. Pharm. Biomed. Anal. 43, 428-434. doi: 10.1016/j.jpba.2006.07.013

Cherdshewasart, W., and Sutjit, W. (2008). Correlation of antioxidant activity and major isoflavonoid contents of the phytoestrogen-rich Pueraria mirifica and Pueraria lobata tubers. Phytomedicine 15, 38-43. doi: 10.1016/j.phymed.2007.07.058 
Falcone Ferreyra, M. L., Rius, S. P., and Casati, P. (2012). Flavonoids: biosynthesis, biological functions, and biotechnological applications. Front. Plant Sci. 3:222. doi: 10.3389/fpls.2012.00222

Ferreyra, M. L. F., Rodriguez, E., Casas, M. I., Labadie, G., Grotewold, E., and Casati, P. (2013). Identification of a bifunctional maize Cand O-Glucosyltransferase. J. Biol. Chem. 288, 31678-31688. doi: 10.1074/jbc.M113.510040

Follak, S. (2011). Potential distribution and environmental threat of Pueraria lobata. Cent. Eur. J. Biol. 6, 457-469. doi: 10.2478/s11535-0100120-3

Fu, L., Niu, B., Zhu, Z., Wu, S., and Li, W. (2012). CD-HIT: accelerated for clustering the next-generation sequencing data. Bioinformatics 28, 3150-3152. doi: 10.1093/bioinformatics/bts565

Fullwood, M. J., Wei, C. L., Liu, E. T., and Ruan, Y. (2009). Nextgeneration DNA sequencing of paired-end tags (PET) for transcriptome and genome analyses. Genome Res. 19, 521-532. doi: 10.1101/gr.0749 06.107

Gaines, T. A., Lorentz, L., Figge, A., Herrmann, J., Maiwald, F., Ott, M. C., et al. (2014). RNA-Seq transcriptome analysis to identify genes involved in metabolism-based diclofop resistance in Lolium rigidum. Plant J. 78, 865-876. doi: $10.1111 /$ tpj.12514

Grabherr, M. G., Haas, B. J., Yassour, M., Levin, J. Z., Thompson, D. A., Amit, I., et al. (2011). Full-length transcriptome assembly from RNA-Seq data without a reference genome. Nat. Biotechnol. 29, 644-652. doi: 10.1038/ nbt. 1883

Graham, T. L. (1991). Flavonoid and isoflavonoid distribution in developing soybean seedling tissues and in seed and root exudates. Plant Physiol. 95, 594-603. doi: 10.1104/Pp.95.2.594

Han, R., Takahashi, H., Nakamura, M., Bunsupa, S., Yoshimoto, N., Yamamoto, H., et al. (2015). Transcriptome analysis of nine tissues to discover genes involved in the biosynthesis of active ingredients in Sophora flavescens. Biol. Pharm. Bull. 38, 876-883. doi: 10.1248/bpb.b14-00834

He, X., Blount, J. W., Ge, S., Tang, Y., and Dixon, R. A. (2011). A genomic approach to isoflavone biosynthesis in kudzu (Pueraria lobata). Planta 233, 843-855. doi: 10.1007/s00425-010-1344-1

He, X. Z., Li, W. S., Blount, J. W., and Dixon, R. A. (2008). Regioselective synthesis of plant (iso)flavone glycosides in Escherichia coli. Appl. Microbiol. Biotechnol. 80, 253-260. doi: 10.1007/s00253-008-1554-7

Hong, S. M., Bahn, S. C., Lyu, A., Jung, H. S., and Ahn, J. H. (2010). Identification and testing of superior reference genes for a starting pool of transcript normalization in Arabidopsis. Plant Cell Physiol. 51, 1694-1706. doi: $10.1093 / \mathrm{pcp} / \mathrm{pcq} 128$

Jung, W., Yu, O., Lau, S. M., O’keefe, D. P., Odell, J., Fader, G., et al. (2000). Identification and expression of isoflavone synthase, the key enzyme for biosynthesis of isoflavones in legumes. Nat. Biotechnol. 18, 208-212. doi: $10.1038 / 72671$

Kawaguchi, R., and Bailey-Serres, J. (2005). mRNA sequence features that contribute to translational regulation in Arabidopsis. Nucleic Acids Res. 33, 955-965. doi: 10.1093/Nar/Gki240

Kerscher, F., and Franz, G. (1987). Biosynthesis of vitexin and isovitexin enzymatic synthesis of the $C$-glucosylflavones vitexin and isovitexin with an enzyme preparation from Fagopyrum esculentum m. seedlings. Z. Naturforsch. 42, 519-524

Keung, W. M., and Vallee, B. L. (1998). Kudzu root: an ancient Chinese source of modern antidipsotropic agents. Phytochemistry 47, 499-506. doi: 10.1016/S0031-9422(97)00723-1

Li, R., Yu, C., Li, Y., Lam, T. W., Yiu, S. M., Kristiansen, K., et al. (2009). SOAP2: an improved ultrafast tool for short read alignment. Bioinformatics 25, 1966-1967. doi: 10.1093/bioinformatics/btp336

Lindgren, C. J., Castro, K. L., Coiner, H. A., Nurse, R. E., and Darbyshire, S. J. (2013). The biology of invasive alien plants in Canada. 12. Pueraria montana var. lobata (Willd.) Sanjappa \& Predeep. Can. J. Plant Sci. 93, 71-95. doi: 10.4141/Cjps2012-128

Miadokova, E. (2009). Isoflavonoids - an overview of their biological activities and potential health benefits. Interdiscip. Toxicol. 2, 211-218. doi: 10.2478/v10102009-0021-3
Miyazawa, M., Sakano, K., Nakamura, S., and Kosaka, H. (2001). Antimutagenic activity of isoflavone from Pueraria lobata. J. Agric. Food Chem. 49, 336-341. doi: 10.1021/jf000255w

Muranaka, T., and Saito, K. (2013). Phytochemical genomics on the way. Plant Cell Physiol. 54, 645-646. doi: 10.1093/Pcp/Pct058

Ramilowski, J. A., Sawai, S., Seki, H., Mochida, K., Yoshida, T., Sakurai, T., et al. (2013). Glycyrrhiza uralensis transcriptome landscape and study of phytochemicals. Plant Cell Physiol. 54, 697-710. doi: 10.1093/pcp/ pct057

Saito, K. (2013). Phytochemical genomics - a new trend. Curr. Opin. Plant Biol. 16, 373-380. doi: 10.1016/j.pbi.2013.04.001

Saito, K., Yonekura-Sakakibara, K., Nakabayashi, R., Higashi, Y., Yamazaki, M., Tohge, T., et al. (2013). The flavonoid biosynthetic pathway in Arabidopsis: structural and genetic diversity. Plant Physiol. Biochem. 72, 21-34. doi: 10.1016/j.plaphy.2013.02.001

Schmittgen, T. D., and Livak, K. J. (2008). Analyzing real-time PCR data by the comparative $\mathrm{C}(\mathrm{T})$ method. Nat. Protoc. 3, 1101-1108. doi: 10.1038 /nprot.2008.73

Serres-Giardi, L., Belkhir, K., David, J., and Glemin, S. (2012). Patterns and evolution of nucleotide landscapes in seed plants. Plant Cell 24, 1379-1397. doi: 10.1105/tpc.111.093674

Simpson, J. T., Wong, K., Jackman, S. D., Schein, J. E., Jones, S. J., and Birol, I. (2009). ABySS: a parallel assembler for short read sequence data. Genome Res. 19, 1117-1123. doi: 10.1101/gr.089532. 108

Steele, C. L., Gijzen, M., Qutob, D., and Dixon, R. A. (1999). Molecular characterization of the enzyme catalyzing the aryl migration reaction of isoflavonoid biosynthesis in soybean. Arch. Biochem. Biophys. 367, 146-150. doi: 10.1006/abbi.1999.1238

Takahashi, H., Morioka, R., Ito, R., Oshima, T., Altaf-Ul-Amin, M., Ogasawara, N., et al. (2011). Dynamics of time-lagged gene-to-metabolite networks of Escherichia coli elucidated by integrative omics approach. OMICS 15, 15-23. doi: 10.1089/omi.2010.0074

Tarazona, S., Garcia-Alcalde, F., Dopazo, J., Ferrer, A., and Conesa, A. (2011). Differential expression in RNA-seq: a matter of depth. Genome Res. 21, 2213 2223. doi: $10.1101 / \mathrm{gr} .124321 .111$

Teng, Y., Cui, H., Yang, M., Song, H., Zhang, Q., Su, Y., et al. (2009). Protective effect of puerarin on diabetic retinopathy in rats. Mol. Biol. Rep. 36, 1129-1133. doi: 10.1007/s11033-0089288-2

Tian, A. G., Wang, J., Cui, P., Han, Y. J., Xu, H., Cong, L. J., et al. (2004). Characterization of soybean genomic features by analysis of its expressed sequence tags. Theor. Appl. Genet. 108, 903-913. doi: 10.1007/s00122-0031499-2

Wang, Z., Gerstein, M., and Snyder, M. (2009). RNA-Seq: a revolutionary tool for transcriptomics. Nat. Rev. Genet. 10, 57-63. doi: 10.1038/ nrg2484

Wong, K. H., Li, G. Q., Li, K. M., Razmovski-Naumovski, V., and Chan, K. (2011). Kudzu root: traditional uses and potential medicinal benefits in diabetes and cardiovascular diseases. J. Ethnopharmacol. 134, 584-607. doi: 10.1016/j.jep.2011.02.001

Ye, J., Fang, L., Zheng, H. K., Zhang, Y., Chen, J., Zhang, Z. J., et al. (2006). WEGO: a web tool for plotting GO annotations. Nucleic Acids Res. 34, W293-W297. doi: 10.1093/Nar/Gkl1031

Conflict of Interest Statement: The authors declare that the research was conducted in the absence of any commercial or financial relationships that could be construed as a potential conflict of interest.

Copyright (C) 2015 Han, Takahashi, Nakamura, Yoshimoto, Suzuki, Shibata, Yamazaki and Saito. This is an open-access article distributed under the terms of the Creative Commons Attribution License (CC BY). The use, distribution or reproduction in other forums is permitted, provided the original author(s) or licensor are credited and that the original publication in this journal is cited, in accordance with accepted academic practice. No use, distribution or reproduction is permitted which does not comply with these terms. 